\title{
Assessment of core activities and supportive functions for the communicable diseases surveillance system in Khartoum state, Sudan, 2005-2007
}

N. Sahal, ${ }^{1}$ R. Reintjes, ${ }^{2,3}$ E.M. Eltayeb ${ }^{4}$ and A.R. Aro ${ }^{7}$

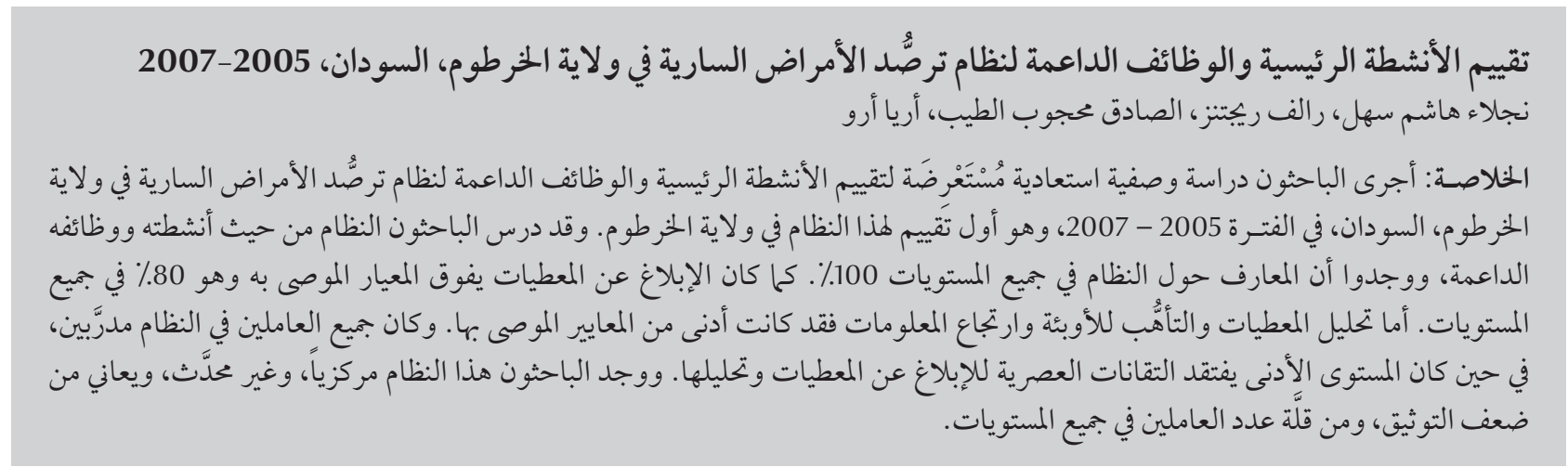

ABSTRACT We conducted a descriptive, retrospective, cross-sectional study to assess the core activities and supportive functions of the communicable diseases surveillance system (CDSS) in Khartoum state, Sudan, for the period 2005-2007. This is the first assessment conducted for CDSS in Khartoum state. The CDSS was studied in terms of core activities and supportive functions. We found that knowledge of the system was $100 \%$ at all levels. Data reporting was over the recommended standard of $80 \%$ at all levels. Data analysis, epidemic preparedness and feedback were below the recommended standard. All assigned CDSS staff members were trained. Lower levels lacked modern technologies for data reporting and analysis. The CDSS system in Khartoum state is centralized; moreover, the system has not been updated, it is poorly documented and has a shortage of staff at lower levels.

Évaluation des activités centrales et des fonctions d'appui du système de surveillance des maladies transmissibles dans l'État de Khartoum (Soudan) de 2005 à 2007

RÉSUMÉ Nous avons conduit une étude descriptive, rétrospective et transversale pour évaluer les activités centrales et les fonctions de soutien du système de surveillance des maladies transmissibles dans l'État de Khartoum (Soudan) de 2005 à 2007. Il s'agit de la première évaluation du système de surveillance des maladies transmissibles dans l'État de Khartoum. Le système de surveillance a été évalué en termes d'activités centrales et de fonctions de soutien. Nous avons observé que la connaissance du système atteignait $100 \%$ à tous les niveaux. La notification des données était supérieure au pourcentage recommandé de $80 \%$ à tous les niveaux également. L'analyse des données, la préparation aux épidémies et le retour d'information étaient insuffisants par rapport aux recommandations. Tous les membres du personnel du système de surveillance des maladies transmissibles en poste avaient reçu une formation. Aux niveaux inférieurs, les technologies modernes faisaient défaut pour la notifification et l'analyse des données. Le système de surveillance des maladies transmissibles de l'État de Khartoum est centralisé. En outre, il souffre d'un retard d'actualisation, d'une documentation médiocre et d'un manque de personnel aux niveaux inférieurs.

'Unit for Health Promotion Research, Institute of Public Health, University of Southern Denmark, Esbjerg, Denmark (Correspondence to N. Sahal: nhsahal@health.sdu.dk).

${ }^{2}$ Department of Public Health, Faculty of Life Sciences, Hamburg University of Applied Sciences, Hamburg, Germany.

${ }^{3}$ Emerging Risks Unit, European Food Safety Authority, Parma, Italy.

${ }^{4}$ Acute Flaccid Paralysis Surveillance Programme, Federal Ministry of Health, Sudan.

Received 13/03/09; accepted: 22/07/09 


\section{Introduction}

Powerful disease surveillance is considered a vital tool for a powerful response system, which is needed to achieve the goal of communicable diseases control [1]. An effective surveillance system has a major role in providing the necessary information for prevention and control of priority communicable diseases; it is considered an important instrument in public health decision-making [1]. The data provided by a surveillance system are important in monitoring the health status of the population and in detecting, preventing and controlling diseases in order to avert major public health problems. Strengthening the disease surveillance and response system is a globally recognized need [2].

Monitoring and evaluation are considered major components of the communicable disease surveillance and response systems; they assist in determining whether the system has achieved its target objectives. Moreover, the results of monitoring and evaluation in terms of outcomes and impact are important for better development of core capacities for the surveillance and response system [3].

Core activities and supportive functions are also considered major components of a communicable disease surveillance system [4]. Core activities are those dealing with case detection, registration, laboratory confirmation, data reporting, data analysis, feedback, and epidemic preparedness and response [4], while, supportive functions are coordination, supervision, training, and mobilization of resources [4].

The communicable diseases surveillance system (CDSS) in Khartoum State was established in 1994 for the collection, analysis and dissemination of communicable diseases data, and is part of the National Surveillance System, also launched in 1994. At first, the system placed emphasis only on malaria with weekly notification from all health facilities using radio stations $(n=107)$. In
January 1999 the system was changed to sentinel sites surveillance (150 sentinel sites), which included 24 hospitals, 91 health centres and dispensaries and 35 nongovernmental organization clinics [5]. The system depends on passive surveillance for communicable diseases, which changes to an active system during epidemics or outbreaks. Communicable diseases such as HIV/AIDS, sexually transmitted infections and tuberculosis have separate surveillance systems outside the integrated CDSS. This leads to an overlap between the systems and to a waste of resources [5].

The data collected are used for planning and monitoring. Hence, there was an urgent need to conduct this study to assess the CDSS and its response capacity to enable the development of a prioritized action plan. The aim of this paper is to assess the core activities and supportive functions of the CDSS in Khartoum state, Sudan, from 2005 to 2007. This is the first assessment conducted for CDSS in Khartoum state as an example for a developing country.

\section{Methods}

A descriptive, cross-sectional, retrospective study design was used. The study population comprised all epidemiology departments/units $(n=177)$ from the 4 levels, state $(n=1)$, locality $(n=7)$, health area $(n=19)$, and health facilities $(n=150)$, participating in the CDSS. A pilot study was conducted in Omdurman locality to test the reliability and validity of the survey. The CDSS core activities (case detection, case registration, case confirmation reporting, data analyses and feedback) and CDSS supportive functions (communication, training, supervision and resources) were measured using World Health Organization (WHO) and Centers of Diseases Control (CDC) standards guide for integrated disease surveillance and response indicators in the African Region [6].
We used a records review survey for data collection for the period 1 January 2005-31 December 2007. At the locality and health area levels, weekly surveillance reports submitted by all health facilities, report tracking tools, case investigation forms, outbreak reports, results of data analysis, epidemic preparedness plans, meeting minutes, schedules and reports for health education and other activities were reviewed. At the health facility level, patient registers, copies of weekly reports, results of data analysis, schedules and reports for community outreach activities, case investigation forms, and standard case definitions were measured. At the state level, weekly reports submitted by all localities were included. The survey was conducted for all CDSS levels using 4 sets of modified generic WHO questionnaires [6].

In 2008 a review of the records at the central CDSS level, locality and health area levels was conducted by a trained medical doctor and health officer, while 14 experts $(7$ medical doctors and 7 health officers) trained in research data collection conducted the review at the health facilities level. The first author, using a sample of the health facilities questionnaire, randomly checked the quality of the reviewers' work. Data from the records review were collected using the standard WHO questionnaire [7]. The analysis was done using SPSS, version 10.0. We used $80 \%$ performance at all CDSS levels as the standard benchmark for each indicator, based on the WHO and CDC guide for Africa [6].

\section{Results}

\section{Presence and objectives of the communicable disease surveillance system}

The system assessed here, the main system for communicable diseases in the state, functioned on different CDSS levels, but there were also 4 parallel, special systems on these levels such as programmes for the prevention and 
control of tuberculosis, leprosy, AIDS and sexually transmitted infections and poliomyelitis. These systems were not completely integrated; instead, they exchanged data with the poliomyelitis surveillance system. The CDSS had clear, specific, written objectives at the central level. However, the degree of clarity was lower moving down the levels of the system.

\section{Assessment of core surveillance and response functions}

\section{Case detection, registration and confirmation}

Case definition is vital for the communicable diseases case detection. A manual of disease-specific case definitions has been distributed to the health facility staff in Khartoum. All staff working at the different CDSS levels knew the diseases under surveillance (Table 1). The capacity for transferring communicable disease specimens was $100 \%$ at lower levels. However, there were no standard written guidelines for specimen collection at any level. About $10 \%$ of the health areas had evidence of followingup or keeping specimen results (Table 1). Central hospitals had capacities to confirm by culture the cases of selected priority diseases. The majority of health facilities, district hospitals and health centres, were not able to perform cultures for any of the priority diseases.

Almost all health facilities had a functioning laboratory, all of which had the ability to collect blood, urine, and stool specimens while less than a quarter were able to collect sputum and cerebrospinal fluid specimens. Almost all laboratories had all the recommended equipment for the collection of blood, urine and stool samples. Over half of the health facilities had the capacity to keep specimens, while less than a quarter were able to transfer the specimen to the reference laboratories (Table 1).

\section{Data reporting, analysis and management}

All health facilities had an outpatient register, and hospitals had an inpatient register for recording of the cases. Data on selected diseases were extracted and reported to the health area level. All CDSS levels had the recommended standard reporting form for the years 2005-2007 (Table 1). All CDSS personnel agreed that reporting was easy and was not time consuming - the average time for preparing the weekly reports was 1 hour at all levels. All CDSS personnel at the lower levels, i.e. locality, health area and health facility levels, were trained in preparing the communicable diseases surveillance weekly reports.

Existence of urgent notification for communicable diseases was found at nearly three quarters of the lower levels, however, there was no evidence that these notifications were sent in the recommended time at all levels (Table 1). Further, there was no evidence of the zero reporting system at lower CDSS levels except in 1 health area. All levels used the standard format for weekly CDSS reports, made at the state level, and all lower levels kept copies of the weekly CDSS reports.

No analysis of communicable diseases surveillance data was done at the health facilities level, and little was done at other lower levels. All lower levels except health facilities had computers for data management (Table 1). All health facilities recorded and processed their data manually. All localities had an epidemic threshold for the priority diseases such as meningitis, malaria and measles, while health areas had a threshold only for meningitis, and none of the health facilities had an epidemic threshold for priority diseases such as meningitis, malaria and measles.

\section{Epidemic preparedness and response}

A case investigation sheet was used by almost all levels. However, there was no evidence on all recommended cases having a special investigation sheet, except for 1 area. None of the lower levels had reports for either the acute watery diarrhea outbreak in 2006 or the rift valley fever outbreak in 2007. The reports for these outbreaks were available at the state level only. None of the lower levels were aware of the number of cases during outbreaks or of the case fatality rates at their level.

None of the lower levels had functioning epidemic management committees for the years we studied as the outbreaks were managed centrally. There was no standard, regular rapid response team at any level; instead, it was activated when needed (Table 1).

\section{Feedback}

At the central CDSS level, all localities and health areas produced a regular feedback report to the lower level (Table 1). There was no standard format for the feedback at lower levels, and none had well formulated feedback.

\section{Communicable diseases surveillance supportive functions}

\section{System guidelines and supervision}

The CDSS had standard guidelines in the form of CDSS manuals, and these were found at the central (state) level and at some of the lower levels (Table 2). However, only about half the lower levels used these guidelines to direct their surveillance activities.

The CDSS had a regular supervision system at all levels. About half of the localities and health areas had performed the recommended supervision visits during the study years (Table 2). All CDSS levels used standard checklists for the supervision. On the other hand, no supervision feedback system existed at lower levels. The system existed from the central to local level in 2005. However, none of the localities was there any evidence of this supervision feedback.

\section{Human resources and communication}

Professional, well-trained staff were available at the central level, whereas at the local level the staff consisted of a medical doctor and a health officer, and at the health area level 1 health officer. Furthermore, the staff at these levels took care of the system as well as 


\begin{tabular}{|c|c|c|c|c|c|c|c|}
\hline \multirow[t]{2}{*}{ Core activity } & \multicolumn{2}{|c|}{$\begin{array}{l}\text { Locality } \\
(n=7)\end{array}$} & \multicolumn{2}{|c|}{$\begin{array}{l}\text { Health area } \\
\quad(n=19)\end{array}$} & \multicolumn{2}{|c|}{$\begin{array}{l}\text { Health facility } \\
\qquad(n=150)\end{array}$} & \multirow[t]{2}{*}{$\begin{array}{l}\text { Standard } \\
\text { benchmark }\end{array}$} \\
\hline & No. & $\%$ & No. & $\%$ & No. & $\%$ & \\
\hline \multicolumn{8}{|l|}{ Case detection } \\
\hline Knowledge of diseases under surveillance & 7 & 100.0 & 19 & 100.0 & 150 & 100.0 & 80 \\
\hline \multicolumn{8}{|l|}{ Case confirmation } \\
\hline Capacity to transport specimens to higher level & 7 & 100.0 & 18 & 94.7 & 26 & 17.3 & 80 \\
\hline Presence of specimen collection guideline & 0 & 0.0 & 0 & 0.0 & 0 & 0.0 & 80 \\
\hline Follow-up of specimen results & 0 & 0.0 & 2 & 10.5 & - & NA & 80 \\
\hline Keeps the specimen result & 0 & 0.0 & 0 & 0.0 & - & NA & 80 \\
\hline \multicolumn{8}{|l|}{ Data reporting } \\
\hline Availability of CDSS reporting form & 7 & 100.0 & 19 & 100.0 & 150 & 100.0 & 80 \\
\hline $\begin{array}{l}\text { Average time to prepare the weekly CDSS report } \\
(1 \mathrm{hr})\end{array}$ & 7 & 100.0 & 19 & 100.0 & 150 & 100.0 & 80 \\
\hline Forward urgent notification for list A diseases & - & NE & - & NE & - & $\mathrm{NE}$ & 80 \\
\hline Submission of urgent notification within $24 \mathrm{hr}$ & - & NE & - & NE & - & NE & 80 \\
\hline Presence of zero reporting system & 0 & 0.0 & 0 & 0.0 & 0 & 0.0 & 80 \\
\hline $\begin{array}{l}\text { Submission of case-based investigation reports } \\
\text { for all recommended cases }\end{array}$ & - & NE & - & NE & - & NE & 80 \\
\hline \multicolumn{8}{|l|}{ Data analysis } \\
\hline Performing trend analysis & 7 & 100.0 & 19 & 100.0 & 0 & 0.0 & 80 \\
\hline Use of appropriate source of denominators & & 0.0 & & 0.0 & & 0.0 & 80 \\
\hline Aggregate case data by demographic category & 7 & 100.0 & 19 & 100.0 & 150 & 100.0 & 80 \\
\hline \multicolumn{8}{|l|}{ Epidemic preparedness and response } \\
\hline Involved in an outbreak investigation & 7 & 100.0 & 19 & 100.0 & 0 & 0.0 & 80 \\
\hline $\begin{array}{l}\text { Implementation of community prevention and } \\
\text { control measures based on local data }\end{array}$ & 0 & 0.0 & 0 & 0.0 & 0 & 0.0 & 80 \\
\hline $\begin{array}{l}\text { Presence of written epidemic preparedness and } \\
\text { response plan }\end{array}$ & 0 & 0.0 & 0 & 0.0 & 0 & 0.0 & 80 \\
\hline $\begin{array}{l}\text { Presence of emergency stocks of drugs and } \\
\text { supplies }\end{array}$ & 0 & 0.0 & 0 & 0.0 & 0 & 0.0 & 80 \\
\hline Existence of epidemic management committee & 0 & 0.0 & 0 & 0.0 & 0 & 0.0 & 80 \\
\hline Presence of health education material & 0 & 0.0 & 0 & 0.0 & - & NA & 80 \\
\hline Existence of vaccination strategy & 7 & 100.0 & 19 & 100.0 & - & NA & 80 \\
\hline Presence of epidemic rapid response team & 0 & 0.0 & 0 & 0.0 & - & NA & 80 \\
\hline Performance of mass vaccination campaign & 7 & 100.0 & 19 & 100.0 & - & NA & 80 \\
\hline Calculation of vaccination coverage & 7 & 100.0 & 19 & 100.0 & - & NA & 80 \\
\hline \multicolumn{8}{|l|}{ Feedback } \\
\hline Received feedback from a higher level & 7 & 100.0 & 19 & 100.0 & 150 & 100.0 & 80 \\
\hline Feedback seen as beneficial & 1 & 14.3 & 12 & 63.2 & 10 & 10.0 & 80 \\
\hline
\end{tabular}

$N A=$ not applicable $; E=$ no evidence.

other heavy duties for other preventive medicine departments. At the health facility level the system had only 1 trained staff member conducting surveillance among other duties. Almost all CDSS staff at all levels were trained in communicable diseases surveillance (Table 2).
About $90 \%$ of the health facilities had functioning communication methods (Table 2). The weekly epidemiological reports were sent manually/on paper from all levels except for 1 remote health facility, which sent them by phone.

\section{Discussion}

\section{Objectives and guidelines of CDDS}

CDDS objectives in Khartoum were found to be clear and well documented at the central level only. None of the 


\begin{tabular}{|c|c|c|c|c|c|c|c|}
\hline \multirow[t]{2}{*}{ Supportive functions } & \multicolumn{2}{|c|}{$\begin{array}{l}\text { Locality } \\
(n=7)\end{array}$} & \multicolumn{2}{|c|}{$\begin{array}{l}\text { Health areas } \\
\qquad(n=19)\end{array}$} & \multicolumn{2}{|c|}{$\begin{array}{l}\text { Health facility } \\
\qquad(n=150)\end{array}$} & \multirow{2}{*}{$\begin{array}{c}\begin{array}{c}\text { Standard } \\
\text { benchmark }\end{array} \\
\%\end{array}$} \\
\hline & No. & $\%$ & No. & $\%$ & No. & $\%$ & \\
\hline \multicolumn{8}{|l|}{ CDSS manual } \\
\hline Presence of the CDSS manual & 5 & 71.4 & 14 & 73.7 & 20 & 13.3 & 80 \\
\hline $\begin{array}{l}\text { Use of the CDSS manual to guide the surveillance } \\
\text { activities }\end{array}$ & 3 & 60.0 & 6 & 46.2 & 13 & 65.0 & 80 \\
\hline \multicolumn{8}{|l|}{ Training } \\
\hline Training of the rapid response team & - & NA & - & NA & - & NA & 80 \\
\hline Basic training on CDSS & 7 & 100.0 & 19 & 100.0 & 131 & 87.3 & 80 \\
\hline Post basic training on CDSS & 7 & 100.0 & 19 & 100.0 & & 74.5 & 80 \\
\hline \multicolumn{8}{|l|}{ Supervision } \\
\hline Presence of supervisory visits to the lower level & 3 & 42.9 & 9 & 47.4 & - & NA & 80 \\
\hline Review of CDSS activities during the supervisory visit & 2 & 28.6 & 3 & 15.8 & - & NE & 80 \\
\hline Existence of supervisory visit feedback system & 0 & 0.0 & 0 & 0.0 & 0 & 0.0 & 80 \\
\hline Implementation of supervisory visit recommendation & - & $\mathrm{NE}$ & - & $\mathrm{NE}$ & - & $\mathrm{NE}$ & 80 \\
\hline \multicolumn{8}{|l|}{ Resources } \\
\hline Presence of office & 7 & 100.0 & 16 & 84.2 & 150 & 100.0 & 80 \\
\hline Presence of functioning telephone & 7 & 100.0 & 19 & 100.0 & 134 & 89.3 & 80 \\
\hline Presence of functioning means of transportation & 7 & 100.0 & 14 & 73.7 & & NA & 80 \\
\hline Availability of functioning computer & 7 & 100.0 & 19 & 100.0 & 22 & 14.7 & 80 \\
\hline Availability of functioning photocopier & 0 & 0.0 & 0 & 0.0 & 0 & 0.0 & 80 \\
\hline Availability of functioning spray pump & 1 & 14.3 & 0 & 0.0 & 0 & 0.0 & 80 \\
\hline Availability of disinfection materials & 1 & 14.3 & 0 & 0.0 & 98 & 65.3 & 80 \\
\hline Availability of protection materials & 1 & 14.3 & 0 & 0.0 & 91 & 60.7 & 80 \\
\hline
\end{tabular}

$N A=$ not applicable $; N E=$ no evidence .

localities or health areas had written objectives although most of the respondents at these levels were fully oriented about them. This shows that the system was well established but lacked documentation at the lower levels. In this respect the CDDS in Khartoum was functioning better than other systems (e.g. the Australian system) where the objectives were not clear [8]. Most of the CDSS personnel in Khartoum used the standard state guidelines, developed in 2001, to direct their activities.

\section{Case detection, registration and confirmation}

The lack of the manual for disease specific case definitions in the most of the studied health facilities implies that the case detection quality faces serious problems. On the other hand, continuous supervision visits, which are regularly conducted at different levels, improve this situation. Khartoum state seems to be behind the other states in Sudan, where the CDSS manual was available in all health facilities [9], however, the situation in Khartoum was better than that of Uganda where the system lacked standard case definitions [10].

Another problem found in Khartoum was that the CDSS case definition manual had not been updated since 2001. This means that it did not include new emerging diseases such as SARS and avian influenza. In this respect the situation is similar to that in Mozambique [6]. Although all health facilities had standard patient registries, in line with 8 other states in Sudan [9], in
Khartoum it was not possible to check whether all cases were registered since no system for double checking of the registration was in place.

Almost all sentinel sites had well functioning laboratories and health centres. Whereas peripheral hospitals were capable of confirming only simple cases, the central hospitals were much better in confirming communicable diseases, but still viral diseases were not within their capability and confirmation was done at the state referral laboratory. Only half of the health facilities were capable of keeping the specimens; this affects case confirmation and leads to notification of more suspected cases as well as to overestimation of cases in the state. This was similar to the situation in the other Sudanese states [9]. 


\section{Surveillance data reporting and management}

The cornerstone of the surveillance system, registration and reporting of priority diseases, was well constructed since all CDSS levels in Khartoum state used the standard data reporting form. However, problems such as no update of the standard form since its establishment and manual data reporting, especially at lower levels (as in other Sudanese states [9]), which leads to reduced data accuracy, weaken the system. On the other hand, the CDSS was concerned with reporting of important communicable diseases only and was not overloaded with unnecessary data as has been reported from the Armenian surveillance system [11].

In the integrated disease surveillance strategy the data collected should be analysed and used for action, especially at the health facility level [1]. Poor data analysis at the lower levels in Khartoum actually indicates a centralized system, which leads to the absence of proper scientific interpretation of the collected data. In this respect the situation resembles that of the other Sudanese states [9], South Africa [12] and Mali and Ghana [13]. Continuous, systematic and more detailed analysis of all data reported at lower levels should be done to keep track of the disease situation in the area and to maximize and strengthen CDSS effectiveness at lower levels.

Another failure of the CDSS data analysis in Khartoum, lack of an appropriate denominator for data analysis, e.g. population per area in the lower levels, means that none of the localities or health areas had a clear idea about the true magnitude of the communicable diseases in their area (except for meningitis due to the special programme). This negatively affects the use of surveillance data to perform the recommended actions in time, and it might also affect early detection of epidemics. Similarly, proper and early action for epidemics is hindered by the fact that neither health areas nor health facilities had any epidemic threshold.

\section{Epidemic preparedness and response}

Khartoum state has experienced outbreaks of cholera and Hemorrhagic fever in the period 2005-2007. However, neither regular epidemic management committees nor rapid response teams were found at any level as only during epidemics were meetings and teams arranged, and in most cases there were no records of the meetings. Furthermore, the lower levels of CDSS had no written epidemic management plan, which affects the effectiveness of organized response to outbreaks. In this respect the Khartoum system was weaker than that in the other states of Sudan [9] but similar to those in Mozambique [6] and Ghana [14].

Monitoring and evaluation of the actions taken suffers from the absence of epidemic management documentation at lower levels of the CDSS system. Further, not knowing the defects of the epidemic response system means that the defects cannot be corrected. The problem seems to be common also in other Sudanese states [9]. In addition, the central, state level stockpiling of drugs and vaccines might delay a quick response to epidemics at other levels. This seems to be a common problem in Sudan [9], Mozambique [6] and in most African countries [14]. Additionally, stopping of the regular vaccination campaign for communicable diseases such as meningitis in 2005 breaks the disease prevention chain and will lead to outbreaks in the coming years. This shows that the CDSS (in African countries) lacks proper planning as the cost of epidemics will be much greater than the cost of campaigns.

\section{Feedback, supervision, human resources and training}

It seems that the absence of standardization and regularity of feedback in the CDSS in Khartoum results in half of the CDSS personnel rating it as non-beneficial, as extra workload and a waste of time. In the absence of feedback, regular standardized supervision provides quality checks and job training but it hampers achievement of the recommended goals and is also a waste of resources within CDSS. The problems in this respect seems to be similar to those in other Sudanese states [9] and in Ethiopia [15].

The CDSS system in Khartoum as well as elsewhere in Sudan [9] has welltrained professional staff at the state level. However, in Khartoum the system is facing shortages of staff at lower levels where the staff conduct surveillance activities along witho other preventive medicine activities. High work overload at those levels affects the quality of the CDSS activities. It has been pointed out that participants in the surveillance system should be properly trained for their surveillance tasks through both initial and ongoing in-service training [16]. In this respect the situation in Khartoum is better than in Tanzania [4] and Uganda [10].

Based on our findings, the CDSS in Khartoum state needs to strengthen the core and support functions of surveillance at all levels of the health system. Formulation of clear written objectives for CDSS at all levels should be the first priority. CDSS data are often not adequately analysed or used to evaluate the effectiveness of intervention programmes. Thus, urgent intervention is needed to build an updated, advanced data analysis system, both for routine surveillance and for outbreaks, to make use of the large amount of data collected at different levels. Furthermore, the system should implement proper documentation methods for all the CDSS data collected, mainly for the urgent notification of communicable diseases and outbreaks data as well as for zero reporting. In addition, the surveillance system needs to develop a standard, regular, effective feedback system. The challenge is to respond quickly and properly to epidemics, thus the formation of a standard rapid response team at all levels is the very first step in building effective epidemic preparedness in Khartoum state. 
Strengthening of CDSS supportive functions in Khartoum state is needed. Adequate human resources at lower levels of the surveillance system as well as the creation of an incentive system, which would maintain commitment to CDSS among the personnel, is needed. Provision of supported, documented supervisory visits to the different levels and timely feedback might create additional support to sustain an effective CDSS that guides public health decision-making in Khartoum state.

In conclusion, well-functioning core activities and supportive functions are the basis of the CDSS to achieve its aim in communicable disease prevention and control. The CDSS in Khartoum state is an old system that adopts theidea of integrated communicable diseases surveillance [17]. The system seems to be functioning well as it has clear objectives and guidelines at the state level but it still has many defects and is facing many challenges. Although the system appears decentralized, there are a number of items, such as data analysis and epidemic management, which are centralized. The Khartoum system was poorly documented at the lower levels, the system wasnotupdated, anditlacked a proper feedback system for both data reporting and supervision. The system also faced the problem of staff shortages at lower levels. In addition, epidemic preparedness was centrally organized and was functioning poorly at lower levels. Laboratory capacity was poor at lower levels.

Overall, the existing CDSS in Khartoum state needs to be strengthened with more-effective coordination so that it can work at its optimum capacity to achieve the global goal of prevention and control of communicable diseases.

\section{References}

1. Pan American Health Organization. An integrated approach to communicable disease surveillance. Epidemiological Bulletin, 2000, 21(1):1-4 (http://www.paho.org/English/SHA/ EB_v21n1.pdf, accessed 1 September 2007).

2. Communicable disease surveillance and response systems, a guide to planning. Geneva, World Health Organization, 2006 (WHO/CDS/EPR/LYO/2006.1).

3. Technical review on monitoring and evaluation protocol for communicable disease surveillance and response systems. Geneva, World Health Organization, 2004 (http://whqlibdoc.who. int/hq/2004/WHO_CDS_CSR_LYO_2004.15_eng.pdf, accessed 18 October 2010).

4. Nsubuga Petal.Structure and performance of infectious disease surveillance and response, United Republic of Tanzania, 1998. Bulletin of the World Health Organization, 2002, 80(3):196-203 (http://www.who.int/bulletin/ archives/80(3)196.pdf, accessed 1 September 2007).

5. A report of strategic plan for National Communicable Disease Surveillance in Sudan. Khartoum, Ministry of Health, Epidemiology Department, 1996.

6. Guide for the use of core integrated disease surveillance and response indicators in the African Region. Geneva, World Health Organization, 2005 (http://www.cdc.gov/idsr/files/guide. pdf, accessed 1 September 2008).

7. Protocol for the assessment of national communicable disease surveillance and response systems, annex 12: Generic questionnaires. Geneva, World Health Organization, 2001 (WHO/ CDS/CSR/ISR/2001.2) (http://www.who.int/csr/resources/ publications/surveillance/whocdscsrisr20012a.pdf, accessed 14 October 2010).

8. Miller M et al. Evaluation of Australia's National Notifiable Disease Surveillance System. Communicable Diseases International, 2004, 28:311-323.

9. Mahdi TF. An interventional study to strengthen the national communicable disease surveillance and response systems in Sudan. Khartoum, Khartoum State Ministry of Health (research documents), 2003.
10. Assessment of Infectious Disease Surveillance Uganda. Morbidity and Mortality Weekly Reports, 2000, 49:687-691.

11. Tadesse $\mathbf{W}$ et al. Assessment of the infectious diseases surveillance system of the Republic of Armenia: an example of surveillance in the Republics of the former Soviet Union. BMC Public Health, 2002, 2(3) (http://www.biomedcentral. com/1471-2458/2/3, accessed 14 October 2010).

12. Weber IB, Matjila MJ, Harris BN. Evaluation of the notifiable disease surveillance system in Gauteng Province, South Africa. The Afrihealth post-conference information, 2007 (http://upetd.up.ac.za/thesis/available/etd-07302008141155/unrestricted/dissertation.pdf, accessed 18 October 2010).

13. The implementation of integrated disease surveillance and response in the African and Eastern Mediterranean Regions, 2003. Atlanta, Georgia, Centers for Disease Control and Prevention, 2003 (http://www.cdc.gov/idsr/focus/surv_ sys_strengthening/doc_idsr_implement.pdf, accessed 18 October 2010).

14. Integrated Disease Surveillance and Response. Integrated Disease Surveillance and Response Update Bulletin 2003. Washington DC, Support for Analysis and Research in Africa (SARA) Project, 2003 (http://www.cdc.gov/idsr/focus/advocacy/ policy_briefsENG.pdf, accessed 17 October 2010).

15. Assessment of the national communicable disease surveillance and response system, Ethiopia. Bulletin of the World Health Organization, 2001, 76:9-16.

16. Integrated approach to communicable disease surveillance. Ottawa, Public Health Agency of Canada, 2000 (http://www. phac-aspc.gc.ca/publicat/ccdr-rmtc/00vol26/dr2607eb. $\mathrm{html}$, accessed 14 October 2010).

17. Sahal N, Reintjes R, Aro AR. Communicable diseases surveillance lessons learned from developed and developing countries. Scandinavian Journal of Public Health, 2009, 37(2):187-200. 